\title{
Tactile facilitation of figure reversal in mental imagery
}

\author{
LEONARD BROSGOLE and JOHN S. MALLOZZI \\ St. John's University, Jamaica, New York
}

\begin{abstract}
In the first of three experiments, we found that $26 \%$ of our subjects reversed the mental image of Jastrow's (1900) duck/rabbit figure. We speculated that the remaining $74 \%$ did not perform the reversal in imagination because they were unable to maintain an accurate image of the figure sufficiently long to allow for the reversal to occur. Therefore, in a second experiment, subjects attempted to reverse the memory image of the duck/rabbit, while simultaneously feeling an embossed version of the figure. This resulted in a $66 \%$ reversal rate, suggesting that the haptic form allowed for a greater number of reversals by preventing the mental image of the duck/rabbit from becoming distorted or decaying over time. Because a good portion of these reversals may have been tactual and not imaginal, a fragmented haptic form was used in a third experiment. A 50\% reversal rate was obtained, even though this form could not be reversed by touch alone.
\end{abstract}

Chambers and Reisberg (1985) aimed three experiments specifically at determining whether subjects could reinterpret the memory image of Jastrow's (1900) duck/rabbit figure. Their procedure involved five basic steps. First, to familiarize their subjects with reversible figures, they allowed the subjects to reverse the vase/face, the Necker cube, and the Mach book. Next, the duck/rabbit figure was exposed for $5 \mathrm{sec}$, and the subjects were told to form a mental picture of it, so that they would be able to draw it later. This was followed by the presentation of the chef/dog figure, which was intended to familiarize the subjects with figure reversal based on a reference frame realignment. After successfully reversing the chef/dog, the subjects were requested again to form an image of the duck/rabbit, inspect it, and reconstrue it. Finally, the subjects were asked to draw a picture of the duck/rabbit figure that was originally shown to them and to reverse the drawing. Chambers and Reisberg found that not 1 of 35 subjects was able to reverse the duck/rabbit in imagery, but that every subject was able to reverse his/her own drawing.

There may have been some procedural and data-related problems in Chambers and Reisberg's (1985) study. With regard to methodology, it is entirely possible that their subjects may not have been able to perform the reversal in imagery because they were told to form an image of the $\mathrm{duck} / \mathrm{rabbit}$ for the precise purpose of drawing it at a later point. The intent of remembering it for later reproduction may have interfered with the reversibility of the memory image. In addition, the interpolation of the chef/dog also may have interfered with the front-back reference-frame

We thank Roy Brosgole for suggesting how to emboss the contours of the fragmented figure and for constructing it. We also thank Dawn Opitz and William Iaccino for assisting us in testing subjects. Requests for reprints should be sent to L. Brosgole, Psychology Laboratory, St. John's University, 81-50 Utopia Parkway, Jamaica, NY 11439. realignment that may be necessary for reversing the duck/ rabbit. These procedural problems may explain why not a single subject was able to perform the reversal in imagery. Other problems, however, raise questions about the credibility of the data.

Chambers and Reisberg (1985) reported that some of their subjects were high visualizers and some were artists and architects. It is interesting that not one of these people reported reversing the duck/rabbit figure during its lengthy 5-sec presentation period. In our own pilot research, we had to reduce the exposure time to $2 \mathrm{sec}$, because we were losing far too many subjects. Also, it is surprising that not one artist or architect was able to reinterpret his/her mental image by using some cognitive strategy. We had subjects who would focus on a part of the image, reinterpret it, and build on it to come up with a new construal. So, for example, some of our subjects reported that the bill of the duck could be the horns of a goat, the ears of a dog, or the tail of a fish. Even if they never really saw the alternate construal of the figure, they were able to report one. It is very odd that artists and architects did not employ a similar strategy. Finally, it is almost inconceivable that every subject in Chambers and Reisberg's (1985) study was so proficient artistically as to be able to reinterpret his/her own drawing. We had subjects who could not reconstrue the duck/rabbit stimulus, let alone their own drawings. Accordingly, the following experiments were conducted to determine whether it is possible to reverse ambiguous figures in mental visual imagery.

\section{EXPERIMENT 1}

In this first experiment, we gathered baseline data on the reversal of the duck/rabbit figure in visual imagery. The procedure was similar to that used by Chambers and Reisberg (1985); there was a training session followed 
by a testing session, in which subjects were encouraged to reverse the mental image of the duck/rabbit. However, there were several essential methodological differences between this experiment and Chambers and Reisberg's. The key differences were that we honestly communicated the intent of the study to our subjects, and attempted to create the impression (during training) that it was possible to reverse mental images. In addition, the last training figure presented involved a left-right reference-frame realignment, as does the duck/rabbit figure.

\section{Method}

Subjects. One hundred subjects were run through the entire procedure. They consisted of 40 males and 60 females, ranging in age from 18 to 59 years, with a mean age of 29.6. An additional 26 subjects were dropped from the study because they reversed the duck/rabbit figure perceptually while it was being exposed. These subjects included 10 males and 16 females, with a mean age of 24.2. All subjects signed an informed consent form. None were familiar with reversible figures.

Stimuli. The reversible training figures consisted of the vase/face, old man/rat, and Indian/Eskimo. The latter figure could be seen as either the head of a Native American facing to the left or the entire body of a person in a fur parka facing behind and to the right. The test figure was the duck/rabbit. All figures were drawn in black and oriented horizontally on $8.5 \times 11$ in. white bond paper. The four drawings occupied rectangular areas ranging from 35 to 48.6 in. ${ }^{2}$ The obliquely oriented duck/rabbit fit into an area of 45 in. $^{2}$ (See Figure 1a.)

Procedure. The subjects were told that our purpose was to determine whether people could reverse ambiguous figures in their imaginations. The first procedural step was to familiarize them with reversible figures, and they were shown the vase/face, old man/rat, and Indian/Eskimo, in that order. Each figure was placed on a table in front of the subject, who had to reverse each figure and had unlimited time to do so. $\mathrm{He} / \mathrm{she}$ was allowed to handle the figures, and prompts were given (e.g., examine the contours, tilt the figure clockwise and counterclockwise) to assist the subject in reversing the figure whenever there was any difficulty. Upon reversing a training figure, the subject was asked to form a mental picture of that figure and to alternate between the two construals until the image faded out and alternation was no longer possible. The subject was told to tap on the table with a pen each time a reversal occurred. This enabled us to keep a count of the number of reversals for a total of $90 \mathrm{sec}$, or until the image faded, whichever came first. The training session was followed by a 2-sec exposure of the duck/rabbit. After viewing the figure, the subject was asked how many construals were seen, but was cautioned not to name the figures seen. If two or more construals were seen, the subject was asked to name them. All subjects who imagined two animals facing in opposite directions were dropped from the remainder of the procedure. All others were retained, even if they reported two figures facing in the same direction (e.g., fish and rabbit).

The subjects were then asked to form a mental image of the picture just shown, attend to it, and attempt to reverse it. Subjects who could not perform the reversal were then prompted to examine the contours of the image. If they still could not perform the reversal, they were en-

\section{Duck/Rabbit Figures}

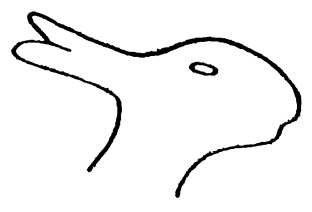

a. Complete

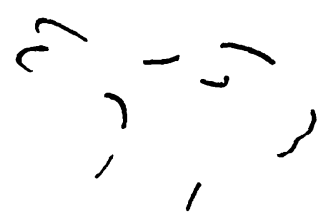

b. Fragmented
Figure 1. Drawing of a complete visual and haptic duck/rabbit (a) and fragmented haptic form (b). couraged to scan the image from left to right and right to left. If they were still unable, they were told that the figure could be seen as the head of an animal facing to the left and the head of a different animal facing to the right. Those who could not perform the reversal even with that prompt were told to try to tilt the image clockwise and counterclockwise. The subjects were given unlimited time with each prompt. Each step of the procedure continued until the subject gave up trying to reverse the image.

The subjects who could not perform the reversal in imagery were then asked to draw a picture of the image and to reverse it. Finally, they were shown the picture of the duck/rabbit once more and were given unlimited time to reverse it.

\section{Results and Discussion}

Twenty-six subjects were able to reverse the duck/rabbit figure in imagery. Seventeen of these reversals followed the reference-frame hint. However, four of the reversals occurred in response to the preceding prompts to inspect the contour and to scan the figure from side to side. An additional 5 people made the reversal subsequent to the reference-frame hint, when told to rotate the picture in imagination.

Of the 26 reversals, 21 were specifically duck/rabbit. Thirteen of these were in response to the reference-frame prompt, 4 to prior prompts, and 4 to the hint that followed. Of the 5 subjects who did not report a duck/rabbit reversal, 1 saw a duck/hamster, 2 saw a duck/fish, 1 a duck/ whale, and 1 a platypus/rabbit.

Ten of the reversals were spontaneous, with one figure suddenly becoming the other. The remaining 16 reversals resulted from subjects cognitively creating a new form, building on one of the parts of the figure being imagined. Thus, a subject who was able to reinterpret the bill of the duck as the ears of a furry animal was likely to report a shift to the head of a rabbit or hamster in imagination.

Seventy-four people were unable to perform the reversal in imagery. Of these, 58 could not reverse their own drawing of the figure being imagined. This suggests that the memory image of the duck/rabbit may have become so distorted as to be nonreversible. It is interesting that 7 subjects could not even reverse the picture of the duck/ rabbit when it was shown at the conclusion of the procedure. This means that only 67 of the 74 nonreversers could have been expected to reverse the duck/rabbit in imagery.

The distribution of reversers and nonreversers (26 vs. 74) differed significantly from that reported by Chambers and Reisberg (1985) $\left(\chi^{2}=11.32, p<.01\right)$. A difference between the two studies still obtained when we omitted the cognitive reconstruals and considered the distribution of 10 spontaneous reversals and 74 nonreversals $\left(\chi^{2}=\right.$ $4.54, p<.05)$. Thus, there has been a failure to uphold the prior findings of Chambers and Reisberg. This shifts the emphasis away from anticipating and accepting nonreversals on theoretical grounds to having to explain why so few subjects were capable of reversing in imagination.

Speculating that there may be a basic difference in imagery ability between reversers and nonreversers, we compared these two groups in terms of the amount of time during which they were able to maintain the training figures in imagination. We also compared both groups on the number of times that they were able to alternate 
imaginally between the two construals of each of the three training figures. No differences were found between the groups on both measures. Yet, the nonreversers complained during the course of the imagery session that the mental image of the duck/rabbit was unclear, vague, and fading, and that only parts of it were present in the imagination. Therefore, the following studies were aimed at assisting subjects in maintaining an accurate mental picture of the duck/rabbit and to determine whether this had a facilitating effect on the frequency of figure reversal.

\section{EXPERIMENT 2}

The procedure used in Experiment 1 was repeated. In order to help subjects properly encode and maintain the mental image of the duck/rabbit figure, one group of subjects was presented with a haptic replica of that figure during the imagery session. These subjects felt the figure (which was out of view) with the dominant hand. They were instructed to follow the imagery hints both imaginally and tactually. A second group was tested to control for the possibility that a disproportionate number of reversals reported by the first group might be due to pure haptic reinterpretations and not reconstruals in imagery. These subjects were presented with the haptic form only. They did not see a picture of the duck/rabbit until the end of the procedure, after they had drawn a picture of what they were touching. Few (if any) reversals were expected tactually, while a moderate number was anticipated with the haptic augmentation of the visual image.

\section{Method}

Subjects. Seventy-two people were tested. They included 33 males and 39 females ranging in age from 17 to 84 , with a mean age of 33.1 . An additional 15 subjects were dropped from the study because they reversed the duck/rabbit perceptually. They included 8 males and 7 females, with a mean age of 31.5. All subjects gave informed consent and none were familiar with reversible figures.

Stimuli. The same stimuli were used as in the previous study. In addition, a replica of the duck/rabbit was cut from .5-in. plywood with a band saw. The eye of the figure was cut completely through the wood. This form was mounted on a piece of .75-in. pine, which was 8.5 in. high $\times 11$ in. wide. The haptic form was concealed in a cardboard box, which had an opening in the front that was 4 in. high $\times 5$ in. wide. The opening was covered by a black felt curtain fastened at the top. Thus, subjects were able to feel (but not see) the form by placing a hand under the felt and through the opening.

Procedure. This was a between-subjects design, with two conditions. In Condition 1, subjects were run through the same procedure as in Experiment 1 , except that the haptic form was presented along with the picture of the duck/rabbit. Prior to presentation of the picture, the subjects were told that they would be shown a reversible figure very briefly and that their task was to reverse it in the imagination. In addition, they were told that they would be given hints to assist them in reversing the picture and that they would be able to feel a wooden cut-out of the figure, which would help them keep the image fixed in the imagination. At the point at which they were asked to form an image of the figure shown, they were directed to place the dominant hand in the box and to explore the haptic form tactually. They were further instructed to use the hand to follow the hints that were given.

In Condition 2, the procedure from Experiment 1 was again followed, except that the subjects were presented with only the haptic figure during the imagery session. Before being presented with the haptic form, the subjects were told that they would be allowed to feel the figure with the dominant hand and that the figure, if seen, could be reversed just as the preceding training figures. Their task was to try to reverse the figure tactually.

\section{Results and Discussion}

In Condition 1, 21 of 32 people (66\%) reversed the figure in imagination. Twelve subjects did so with the reference-frame prompt, and 9 with the prior scanning prompts. Nine of the reversals occurred spontaneously and 12 were cognitive reconstruals. Eighteen of the 21 reversals involved imagining both a duck and a rabbit. Of the 11 nonreversers, all subjects were able to reverse the picture of the duck/rabbit at the end of the procedure, but only 3 were able to reverse their own drawings.

In Condition 2,11 of 40 people $(28 \%)$ reversed the haptic form. Ten of these reversals occurred in response to the reference-frame hint and 1 to a prior prompt. There was 1 spontaneous reversal, and there were 10 cognitive reconstruals. There were only 2 duck/rabbit reports. Of the 29 nonreversals, only 2 consisted of subjects reversing their own drawings; 22 subjects reversed the picture of the duck/rabbit when it was presented at the conclusion of the procedure.

There was a significantly greater rate of reversals than of nonreversals in Condition 1 than in Condition $2\left(\chi^{2}\right.$ $=10.47, p<.01$ ). In addition, there was a significantly greater reversal rate in Condition 1 than in Experiment 1 $\left(\chi^{2}=16.58, p<.01\right)$. However, Condition 2 produced essentially the same results as did Experiment $1\left(\chi^{2}=\right.$ .03 , n.s.).

The results from Condition 1, along with the phenomenological reports of the subjects, indicated that the haptic figure was tremendously instrumental in enabling the subjects to maintain and reverse the image of the duck/ rabbit. The results of Condition 2 were unanticipated, because they were substantively the same as those from Experiment 1 when the duck/rabbit was presented visually. However, the subjects in Condition 2 indicated that they attempted to convert their tactual impressions into a visual counterpart. If this was the case, our subjects may have defeated the intent of our study by actually reversing a visual representation of the haptic form. Therefore, the purpose of the following study was to create a haptic figure that could not be represented visually, unless preceded by an actual picture of the reversible figure.

\section{EXPERIMENT 3}

This time, the haptic figure was replaced by a Street-like haptic version of the duck/rabbit; one that was so fractionated that it could not be identified by touch, unless preceded by the visual stimulus itself. Using precisely the same design as the last study, we expected that this figure would facilitate figure reversal in the first condition only.

\section{Method}

Subjects. Thirty-two people were tested. They included 13 males and 19 females ranging in age from 13 to 36 , with a mean age of 24.3. An additional 19 subjects were dropped from the study because they reversed 
the duck/rabbit perceptually. They included 9 males and 10 females, with a mean age of 26.1. All subjects gave informed consent and none were familiar with reversible figures.

Stimuli. The materials were the same as in Experiment 2, except that the haptic form was replaced by a fragmented version. (See Figure 1b.) The broken contours of this form were .125 in. wide and raised above a flat wooden surface by .125 in. The entire form was made of wood, and the embossed effect was created by routing out the entire surface of the wood, except for informative pieces of contour (e.g., the bill of the duck, the mouth of the rabbit, a portion of the eye).

Procedure. The procedure was exactly the same as in the last study, except that the haptic form was replaced by a fragmented haptic figure.

\section{Results and Discussion}

In Condition 1, 10 of 20 people (50\%) reversed the duck/rabbit figure in imagination. Eight of the 10 reversals involved duck/rabbit reconstruals, and 2 were shifts between a duck and a fish. Five of the reversals were spontaneous, and 5 were cognitive reconstruals. Seven of the 10 reversals occurred in response to the referenceframe hint, and 3 were prompted by the prior hints to scan the figure in imagination.

Of the 10 people who did not perform the reversal in imagination, only 1 reversed his/her drawing. However, all subjects reinterpreted the duck/rabbit figure perceptually when it was presented at the conclusion of the procedure.

There were 12 subjects in Condition 2, and not one reversed the fragmented haptic form. Three people were unable to make any sense of the figure at all. Each of the remaining 9 subjects offered only one interpretation of the form. One report involved a rabbit, and one, a bird. The others included a ram, deer, butterfly, and so forth. Only 1 subject reconstrued his/her own drawing, reversing from a bird to a fish. All subjects reversed the picture of the duck/rabbit when it was presented at the end of the procedure.

There was a significantly greater rate of reversal in Condition 1 than in Condition $2\left(\chi^{2}=8.73, p<.01\right)$. In addition, there was a significantly greater rate of reversal in Condition 1 than in Experiment $1\left(\chi^{2}=4.64, p<\right.$ $.05)$. Finally, the results of Condition 1 did not differ significantly from those obtained in the first condition of Experiment $2\left(\chi^{2}=1.24\right.$, n.s. $)$. Taken together, these findings indicate that figure reversal in imagination was facilitated by allowing the subjects to explore a representation of visual form tactually, even though the haptic figure could not be reversed by touch alone. This suggests that the tactile stimulus was effective in allowing reversals to occur, insofar as it corrected for the faulty encoding of the form and prevented its memory image from fading and becoming distorted with the passage of time.

Our findings are consistent with those of Finke, Pinker, and Farah (1989), who showed that subjects were capable of altering and combining the mental images of different stimuli to produce novel reconstruals in imagination. Our results also agree with the findings of Peterson, Kihlstrom, Rose, and Glisky (1992), whose study was similar to that of Chambers and Reisberg (1985). However, they used the goose/hawk as a training figure and presented it immediately prior to the duck/rabbit. The goose/hawk involved a pure left/right reference-frame realignment. Its use was followed by an $80 \%$ reversal rate of the duck/rabbit in imagination. Finally, Council, Chambers, Jundt, and Good (1991), found a 10\% reversal rate of the duck/rabbit figure when replicating Chambers and Reisberg's original design. This reversal rate may seem small, but it was obtained without the use of reference-frame hints. (Only $4 \%$ of the subjects in our first experiment performed the reversal prior to the reference-frame hints.) Thus, converging evidence disputes the results reported by Chambers and Reisberg, and Chambers herself contributed to that evidence.

Reisberg and Chambers (1991) indicated that referenceframe realignment may be necessary for the reconstrual of ambiguous mental images and that reinterpretation based on the geometry of these images may not be possible. On the contrary, we found that $28 \%$ of our subjects (across all three experiments) reversed the image of the duck/rabbit prior to receiving a reference-frame hint. It would seem that these subjects reconstrued the mental image on the basis of its geometry. Therefore, a referenceframe realignment may be sufficient but not necessary for generating figure reversal. In fact, it could be argued that a reference-frame realignment may be the phenomenal outcome, rather than the cause, of perceptual and imaginal reconstruals.

With regard to the interpretation of our data, even Chambers and Reisberg (1992) concluded that information may be omitted from a mental image (even though they seemed to argue to the contrary in their 1985 study). With respect to the duck/rabbit, the missing information was found to be from the back of the animal's head, thereby precluding figure reversal. It would follow that such missing information, whether due to decay, distortion, or faulty encoding, could be restored by use of a tactile stimulus and that this should enhance the frequency of figure reversal in the imagination. Such thinking was supported by our data.

\section{REFERENCES}

Council, J. R., Chambers, D., Jundt, T. A., \& Good, M. D. (1991) Are the mental images of fantasy prone persons really more "real'? Imagination, Cognition, \& Personality, 10, 319-327.

Chambers, D., \& Reisberg, D. (1985). Can mental images be ambiguous? Journal of Experimental Psychology: Human Perception \& Performance, 11, 317-328.

Chambers, D., \& Reisberg, D. (1992). What an image depicts depends on what an image means. Cognitive Psychology, 24, 145-174.

Finke, R. A., Pinker, S., \& FARAH, M. J. (1989). Reinterpreting visual patterns in mental imagery. Cognitive Science, 13, 51-78.

JASTROW, J. (1900). Fact and fable in psychology. New York: Houghton Mifflin.

Peterson, M. A., Kihlstrom, J. F., Rose, P. M., \& Glisky, M. L. (1992). Mental images can be ambiguous: Reconstruals and referenceframe reversals. Memory \& Cognition, 20, 107-123.

ReISBERG, D., \& ChAmbers, D. (1991). Neither pictures nor propositions: What can we learn from a mental image? Canadian Journal of Psychology, 45, 336-352.

(Manuscript received June 15, 1993.) 